\title{
Congenital atrichia with papular lesions: a rare cause of irreversible childhood alopecia
}

\author{
Tasleem Arif ${ }^{1 凶}$, Mohammad Adil ${ }^{1}$, Syed Suhail Amin ${ }^{1}$
}

\begin{abstract}
Congenital atrichia with papular lesions (APL) is a disease characterized by the complete absence of hair from the whole body occurring within a few months of birth and the presence of papules distributed over the body. A 9-year-old boy presented with sparse hair over his body and with a history of losing his hair soon after birth. Multiple skin-colored papules were observed over the scalp. A biopsy from a papule showed keratinous cysts in the dermis and the absence of hair follicles. A diagnosis of congenital APL was made after vitamin D-dependent rickets was excluded. This case highlights congenital APL as a cause of total alopecia.
\end{abstract}

Keywords: atrichia, keratin cysts, papules, papular lesions

Received: 13 August 2017 | Returned for modification: 3 October 2017 | Accepted: 18 October 2017

\section{Introduction}

Congenital atrichia with papular lesions (APL) is a rare disease with autosomal recessive inheritance characterized by scarring alopecia that develops soon after birth. Later, the disease is characterized by the development of cysts filled with keratin and of papules involving various parts of the body (1). The case presented here is an unusual presentation of this disease, with alopecia and with papules limited only to the scalp.

\section{Case report}

A 9-year-old boy presented with total hair loss over his body, including the scalp, eyebrows, and eyelashes. The child was born of a nonconsanguineous marriage and had sparse hair over his scalp at birth. This hair had shed completely within a month of his birth. The patient started to develop skin-colored raised lesions over his scalp around the age of 3 years. These lesions were gradually progressive and were asymptomatic. The patient had consulted several private practitioners in the past but had received no relief from his complaints. He had attained the developmental milestones usual for his age. The patient was the first of three siblings, and the other two did not exhibit these signs. There was no family history related to his complaints. There was no history of thickening of the palms and soles, decreased sweating, problems with vision or hearing, seizures, or bone pain.

An examination of the patient revealed very sparse hair over his scalp, eyebrows, eyelashes, and elsewhere on his body (Fig. 1). He was found to have multiple skin-colored papules of around 1 to $3 \mathrm{~mm}$ in size present over his scalp, predominantly over the vertex. The rest of the skin-including the palms and soles, nails, teeth, and mucosae-was normal. The patient's physical growth was normal for his age. No bony abnormalities were present on musculoskeletal examination. Routine laboratory investigations, including complete blood counts (CBC), kidney function tests (KFT), and liver function tests (LFT), were unremarkable. The 1,25 dihydroxycholecalciferol level was found to be $39.6 \mathrm{ng} / \mathrm{ml}$ (the normal range is 30 to $75 \mathrm{ng} / \mathrm{ml}$ ). The patient's serum levels of copper and ceruloplasmin were normal. Radiographs of both wrists were normal. A differential diagnosis of congenital APL, vitamin D-dependent rickets, ectodermal dysplasia, and alopecia universalis was considered. A punch skin biopsy from the scalp revealed the absence of hair follicles, keratin-containing cysts in the dermis, and the absence of inflammatory cells (Fig. 2). Based on the patient's history and the suggestive clinical examination-and further supported by the histopathologic findings-a diagnosis of congenital APL was made.

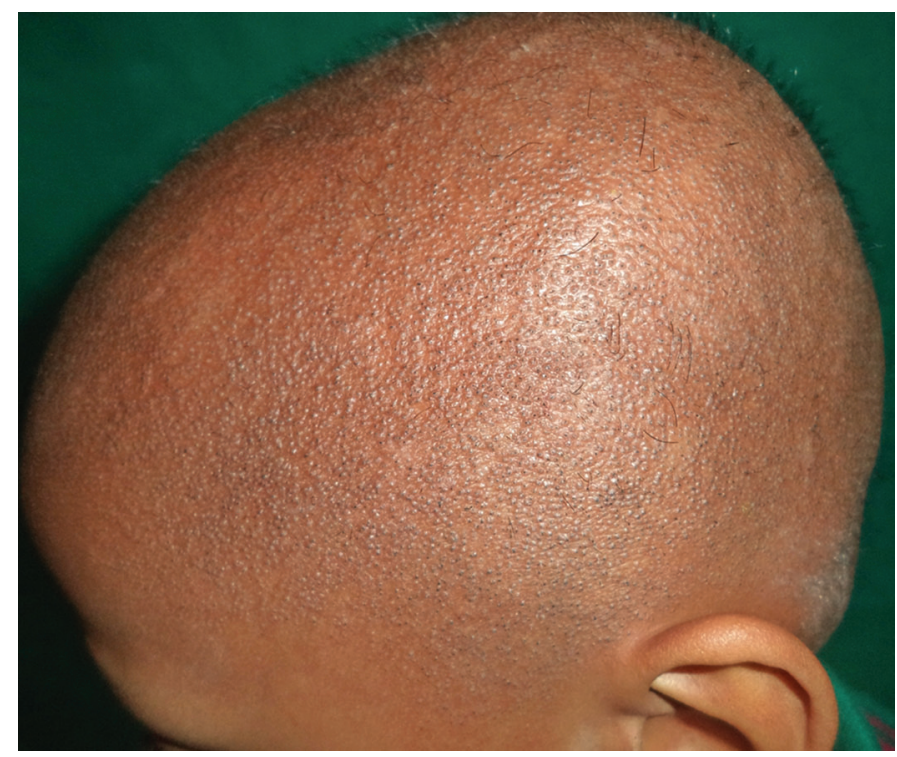

Figure 1 | Diffuse hair loss with generalized papular lesions involving the scalp.

\section{Discussion}

Congenital APL is one of the rare causes of generalized alopecia and is caused by a mutation in the human hairless gene, which is situated on chromosome 8 (short arm) (2). A loss of messaging between the stem cells of the bulge area and the cells of the dermal papilla occurs due to the massive apoptosis of the hair matrix and a decrease in Bcl-2 expression, stranding the papillae in the dermis and leading to the cessation of hair growth (3). The disease is known to be common among an ethnic minority in Ireland called the Irish Travelers (4). 

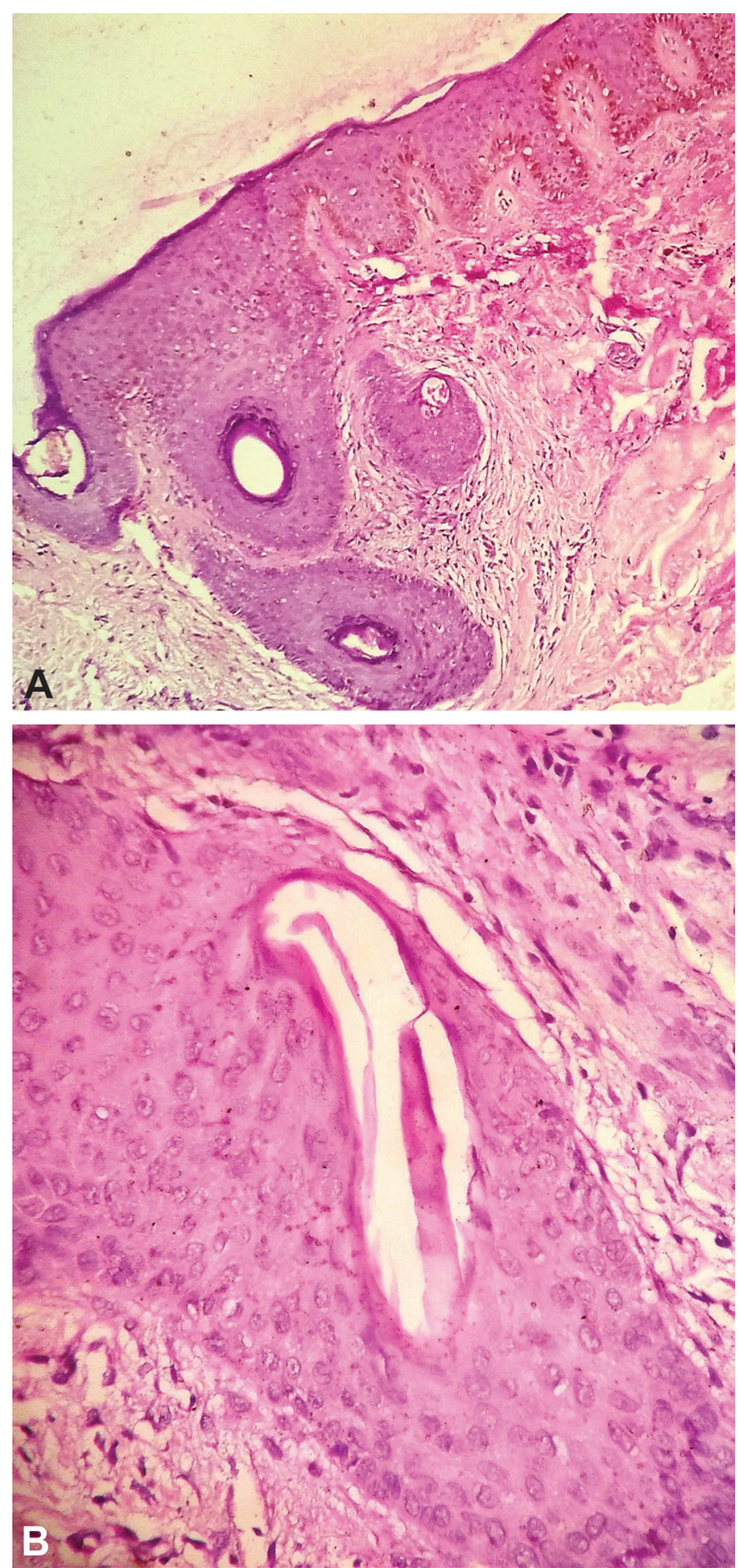

Figure 2 | Histopathology of a papular lesion from the scalp showing (a) multiple keratinous cysts in the superficial dermis (H\&E stain 10x) and (b) a hair follicle with an absent hair shaft (H\&E 40x).

\section{References}

1. Verma R, Vasudevan B, Pragasam V, Badad A, Mitra D, Neema S. Congenital atrichia with papular lesions. Indian J Paediatr Dermatol. 2014;15:94-5.

2. Robati RM, Zereh Posh FB, Sakoei S, Ranjbar M. Unusual presentation of atrichia with papular lesions. Indian J Dermatol. 2015;60:107.

3. Thomas M, Daniel S. Atrichia congenita with papular lesions. Indian J Dermato Venereol Leprol. 2011;77:70-2.

4. Ahmad W, Panteleyev AA, Christiano AM. The molecular basis of congenital atrichia in humans and mice: mutations in the hairless gene. J Invest Dermatol Symp Proc. 1999;4:240-3.

5. Loffreda DM. Inflammatory diseases of hair follicle, sweat glands and cartilage. In: Elder DE, Elenitsas R, Johnson BL, Murphy GF, editors. Lever's Histopathology of the skin. 10th ed. Philadelphia: Lippincott Williams and Williams; 2009. p. 490.
Congenital APL is characterized by hair loss that occurs shortly after birth and the formation of keratin-filled cysts over the body, usually appearing around the age of 2 years. There is no abnormality of nails, teeth, sweating, or hearing, which was true of the case presented here. The histopathologic report of our case was consistent with the expected histopathology of congenital APL: the infundibular portions of the hair follicles were present, but the middle and lower portions were replaced by keratinizing cysts (5).

Yip et al. have proposed a revision of the diagnostic criteria for congenital APL. The proposed criteria include five major and five minor criteria, and at least four major criteria are required for diagnosis. The major criteria are as follows: 1) the permanent and complete absence of scalp hair within the first few months of life, 2) the presence of few to widespread smooth, whitish, or milialike papules on the face, scalp, arm, eyebrows, thighs, or knees since infancy or childhood, 3) the replacement of mature hair follicular structures by follicular cysts filled with cornified material in scalp histology, 4) mutations in the human hairless gene identified by genetic testing, and 5) the clinical and/or molecular exclusion of vitamin D-dependent rickets. The minor criteria are as follows: 1) a family history of consanguinity, 2) the absence of secondary axillary, pubic, or body hair growth and/or sparse eyebrows and eyelashes, 3) normal growth and development, including normal bones, teeth, nails, and sweating, 4) whitish hypopigmented streaks on the scalp, and 5) lack of response to any treatment modality (6). The patient whose case is presented here satisfied four of the five major criteria and three of the five minor ones. Genetic testing was not done due to a lack of resources and the unavailability of the facility. It is important to note that a phenotype similar to APL is seen in vitamin D-dependent rickets, in which a mutation occurs in the vitamin D receptor gene (7). Alopecia universalis is another differential diagnosis. Congenital APL has been associated with several conditions, such as hidrotic ectodermal dysplasia, Moynahan's syndrome, situs inversus, and premature ageing syndromes (8). However, no such association was noted in the case presented here. Another feature that made this an unusual case is that the papules were localized to the scalp and did not involve other parts of the body.

\section{Conclusion}

When dealing with cases of universal alopecia resistant to therapy and associated with papules, it becomes imperative to consider a possible diagnosis of congenital APL. The case presented here was unique because the papular lesions were confined only to the scalp.
6. Yip L, Horev L, Sinclair R, Zlotogorski A. Atrichia with papular lesions: a report of three novel human hairless gene mutations and a revision of diagnostic criteria. Acta Derm Venereol. 2008;88:346-9.

7. Bansal M, Manchanda K, Lamba S, Pandey SS. Atrichia with papular lesions. Int J Trichology. 2011;3:112-4.

8. Sacchidanand S, Sahana M, Hiremagalore R, Asha G. Congenital atrichia associated with situs inversus and mesocardia. Int J Trichology. 2012;4:181-3. 